\title{
Nota Farmacológica: ¿Cuál es la evidencia para usar estimulantes del sistema nervioso central en niños con déficit de atención e hiperactividad?
}

\author{
What is the evidence for using CNS stimulants to treat ADHD in children?
}

Traducido con autorización de "Therapeutics Initiative" (The University of British Columbia). Disponible en URL: http://www.ti.ubc.ca/PDF/69.pdf

Exponer a los niños a medicamentos por períodos prolongados es un tema de preocupación tanto para los padres como para los médicos. Una revisión reciente de la base de datos de medicamentos "Pharmanet" de la región canadiense de British Columbia reveló que el metilfenidato (Ritalin ${ }^{\mathrm{R}}$, Ritalin $\mathrm{SR}^{\mathrm{R}}$, Biphentin $^{R}$, Concerta ${ }^{\mathrm{R}}$ ) es el medicamento al cuál están expuestos en forma crónica (más de dos años) con mayor frecuencia los niños. El metilfenidato se encuentra clasificado dentro de los estimulantes del sistema nervioso central (SNC). Otros estimulantes son la dextroanfetamina (d-anfetamina, Dexedrine $^{\mathrm{R}}$, Dexedrine $\mathrm{SR}^{\mathrm{R}}$ ) y las sales anfetamínicas mixtas (d-anfetamina y l-anfetamina, Aderal $\mathrm{XR}^{\mathrm{R}}$ ). Estos medicamentos son prescriptos generalmente a niños con una gama de problemas de atención y comportamiento denominado síndrome de déficit de atención e hiperactividad (SDAH).

Si bien la revisión de este controvertido y complejo síndrome y su terapéutica, escapan a los alcances de esta nota; este artículo resume y critica la evidencia surgida a partir de los ensayos clínicos publicados, que han sido claves en relación al uso de estimulantes del SNC en niños. Presentamos los beneficios y riesgos surgidos de ensayos clínicos con seguimiento corto (menor a un año) mediano (uno a dos años) y prolongado (mayor a dos años).

Los principios del uso racional y las implicancias clínicas de tratar niños con estimulantes del SNC son relevantes para cualquier droga utilizada durante el período de desarrollo de los seres humanos. Es posible que se produzcan efectos adversos potencialmente irreversibles en relación al crecimiento y desarrollo ligados al uso de fármacos, y los beneficios y daños pueden no manifestarse hasta mucho tiempo después de su utilización. Es por estas razones que ,al pensar en niños, resulta particularmente relevante contar con información surgida de Ensayos Clínicos Controlados Aleotorizados con seguimiento prolongado, sobre el balance entre los beneficios y daños potenciales de los medicamentos ${ }^{1}$

\section{La mejor evidencia de corto plazo (menos de un año) disponible \\ Beneficios}

Muchos ECCA han analizado la eficacia a corto plazo de los estimulantes del SNC en niños. Aunque la Colaboración Cochrane no cuenta con una revisión propia sobre el tema, sí provee 13 referencias a otras revisiones sistemáticas relacionadas. Las dos más relevantes para esta nota terapéutica son las de Schachter y $\mathrm{col}^{2}$. y la de King y $\mathrm{col}^{3}$.

Schachter identificó 62 ECCA publicados entre 1981 y 1999 que habían comparado metilfenidato y placebo, y que incluyeron 2897 participantes (88\% de sexo masculino) con una edad promedio de nueve años. Los ECCA fueron pequeños (tamaño muestral promedio 47) utilizaron un diseño de entrecruzamiento (84\%) y fueron breves (duración promedio del tratamiento tres meses). Los revisores meta-analizaron puntajes de hiperactividad realizados por padres y maestros a partir de un índice de 0 a 31 puntos que medía hiperactividad o comportamiento disruptivo (a menor menor hiperactividad, o mejor resultado del tratamiento).

El tamaño del efecto del metilfenidato a partir de la evaluación de maestros fue de seis puntos de disminución en la escala de hiperactividad partiendo de 14; y a partir de la evaluación de padres fue de cuatro puntos de disminución partiendo de 14. De todas maneras, Schachter y col. consideraron que este tamaño del efecto podía haber estado sobreestimado debido al sesgo de publicación (no publicar ECCA con resultados negativos) y a efectos más fuertes en ECCA con mecanismos de ceguera inadecuados.

King y col. revisaron 65 ECCA hasta julio del 2004, tanto controlados con placebo como ensayos que habían comparado cabeza a cabeza metilfenidato con d-anfetamina. Los revisores resumieron los resultados pero no realizaron un meta-análisis de los datos. De forma similar a Sachter y col, notaron un reporte inadecuado de la metodología de los estudios, posibles sesgos, confiabilidad limitada de los resultados y reporte inadecuado de eventos adversos. Concluyeron que los estimulantes del SNC reducen los puntajes de hiperactividad de padres y maestros, sin diferencias significativas entre ambas drogas, tanto en eficacia como en efectos adversos, debido principalmente a falta de evidencia.

\section{Daños}

La revisión de Sachter y col. incluyó diez ECCA que reportaron eventos adversos ${ }^{2}$. El análisis de los resultados mostró un aumento atribuible a metilfenidato estadísticamente significativo de los siguientes eventos adversos: disminución del apetito (NND o número necesario a tratar para causar un evento adverso $=4)$ insomnio $(N N D=7)$ cefalea $(N N D=22)$ dolor abdominal $(N N D=9)$ y mareos $(N N D=11)$.

La mejor evidencia a mediano plazo (uno a dos años) disponible

Beneficios

La mejor evidencia sobre la eficacia a mediano plazo de los estimulantes del SNC surge del ECCA denominado "MTA" o tratamiento multimodal comparado activo para niños con SDAH, realizado por el Instituto Nacional de Salud Mental de Estados Unidos, que duró 14 meses $^{4}$. Este estudio aleatorizó 579 niños de siete a nueve años de edad a una de cuatro ramas abiertas: sólo estimulantes del SNC (principalmente metilfenidato) sólo terapia conductual, combinación de las dos ramas previas, o tratamiento usual en la comunidad (generalmente tratamiento con estimulantes del SNC).

Los maestros, que no eran ciegos a la intervención, calificaron a los participantes a los 14 meses con un puntaje de hiperactividad/impulsividad que iba de cero a tres puntos. Los resultados de dicha evaluación fueron significativamente mejores en los niños pertenecientes a los dos grupos que habían recibido estimulantes del SNC ( 0,8 puntos) en comparación con los que habían sido asignados a terapia conductual (1,1 puntos) y a tratamiento usual (1,3 puntos). Esta diferencia se mantuvo a los 14 meses del estudio.

Sin embargo, la calificación de observadores "ciegos" a la intervención adjudicada, que se encontraban dentro de las aulas no mostró diferencias entre los cuatro grupos. Además, tampoco se observaron diferencias en los puntajes de la escala multidimensional de ansiedad que los propios niños realizaron.

Respecto de las medidas de desempeño académico -como el Test de Desempeño Individual de Wechsler, que incluye dominios de lectura, matemática y deletreo, con un promedio de 100 (rango 40-160) de acuerdo a la edad- la mayoría de las mediciones tampoco fueron significativamente diferentes, con la única excepción del dominio de lectura, en la que el grupo de terapia combinada obtuvo un puntaje mayor (99) que el de terapia conductual (96) y el de tratamiento usual (95).

\section{Daños}

Se realizó un monitoreo mensual no ciego de eventos adversos en las dos ramas de estimulantes del SNC. Al final del estudio, 
$49,8 \%$ reportó eventos adversos leves, $11,4 \%$ moderados y 2,9 $\%$ severos.

\section{La mejor evidencia a largo plazo (más de dos años) disponible \\ Beneficios}

No hay ECCA que hayan evaluado en niños los riesgos y los beneficios de la terapia a largo plazo con estimulantes del SNC. El único trabajo aleatorizado que incluyó un seguimiento a largo plazo proviene de una extensión del estudio "MTA" descripto previamente. De los 579 niños aleatorizados, 485 $(84 \%)$ fueron seguidos y sus datos re-analizados a los tres $a^{n}{ }^{5}$. A los tres años, tomaba medicación más del $50 \%$ del tiempo transcurrido el $45 \%$ de los niños asignado a terapia conductual, el $72 \%$ de los asignados a tratamiento con estimulantes, el $70 \%$ de los asignados a tratamiento combinado y el $62 \%$ del los asignados a "tratamiento usual". A los tres años fueron medidos nuevamente cinco puntos finales que habían sido estadísticamente diferentes cuando habían sido evaluados a los 14 meses y no hubo diferencias estadísticamente significativas para ninguno de ellos. Además, a los tres años sólo el $50 \%$ de los niños continuaba con criterios diagnósticos de $\mathrm{SDAH}$, sin diferencias entre los cuatro grupos.

\section{Daños}

A los tres años de seguimiento, las tasas de incidencia de delincuencia y abuso de sustancias no difirieron entre los grupos. La tasa global de delincuencia fue de $27 \%$-no se reportaron tasas de delincuencia discriminadas por grupos-, mientras que la tasa de abuso de sustancias fue $22 \%$ para los asignados a estimulantes, $19 \%$ para los asignados a tratamiento usual, $16 \%$ para los asignados a tratamiento combinado y $13 \%$ para los asignados a tratamiento conductual ${ }^{6}$. También se eva- luó el efecto de los estimulantes en la velocidad de crecimiento. En este sentido y en promedio, los niños que estuvieron medicados durante todo el tiempo, durante y después del MTA $(n=70)$ crecieron $3 \mathrm{~cm}$ menos de talla y pesaron $2,7 \mathrm{~kg}$ menos que los niños nunca medicados $(n=65)$ durante esos 3 años ${ }^{7}$.

\section{Conclusiones}

En niños con diagnóstico de SDAH los estimulantes del SNC: - Mejoran los puntajes de los maestros y los padres en las escalas de comportamiento disruptivo por hiperactividad e impulsividad.

- No mejoran los puntajes de ansiedad de los propios niños ni sus logros académicos.

- No varían la incidencia de delincuencia o abuso de sustancias a los tres años de seguimiento.

- No se han estudiado sus efectos a largo plazo sobre los exámenes estandarizados, calidad de vida, tasa de finalización de estudios, empleo, longevidad y salud futura.

\section{Recomendaciones}

Se necesitan grandes ECCA que sigan hasta la edad adulta cohortes de niños tratados con estimulantes del SNC y no tratados, para poder evaluar la incidencia de delincuencia, abuso de sustancias, logros académicos -exámenes estandarizados, años completados de escolaridad, etc.- empleo o desempeño económico y morbi-mortalidad a largo plazo ${ }^{1}$

Antes de poder recomendar el uso a largo plazo de estimulantes del SNC en niños con SDAH es necesario contar con mejor evidencia sobre sus beneficios y riesgos.

La versión preliminar de esta nota terapéutica fue enviada para su revisión por 54 expertos y médicos de atención primaria para corregir cualquier inexactitud y asegurar que la información fuera concisa y relevante.

\section{Referencia}

1. Klassen TP, Hartling L, Craig JC, Offringa M. Children are not just small adults: The urgent need for high-quality trial evidence in children. PLoS Med 5(8):e172.doi:10.1371/journal.pmed.0050172.

2. Schachter HM, Pham B, King J, et al. How efficacious and safe is short-acting methylphenidate for the treatment of attention-deficit disorder in children and adolescents? A meta-analysis. CMAJ. 2001:165:1475-1488.

3. King S, Griffin S, Hodges Z, et al. A systematic review and economic model of the effectiveness and cost-effectiveness of methylphenidate, dexamfetamine and atomoxetine for the treatment of attention deficit hyperactivity disorder in children and adolescents. Health Technol Assess 2006;10(23):iii-iv, xiii-146.

4. The MTA Cooperative Group. A 14-month randomised clinical trial of treatment strategies for attention-deficit/hyperactivity disorder. Arch Gen Psychiatry 1999;56:1073-1086.

5. Jensen PS, Arnold LE, Swanson JM et al. 3-year follow-up of the NIMH MTA

study. J Am Acad Child Adolesc Psychiatry 2007 46:989-1002.

6. Molina BS, Flory K, Hinshaw SP et al. Delinquent behavior and emerging substance use in the MTA at 36 months: prevalence, course, and treatment

effects. J Am Acad Child Adolesc Psychiatry. 2007:46:1028-40.

7. Swanson JM, Elliott GR, Greenhill LL et al. Effects of stimulant medication

on growth rates across 3 years in the MTA follow-up. J Am Acad Child Adolesc Psychiatry. 2007;46:1015-27.

La nota terapéutica presenta un resumen de la evidencia obtenida de ensayos clínicos farmacológicos evaluados críticamente, que puede aplicarse a pacientes similares a los incluídos en esos ensayos pero no ser generalizable a cada paciente en particular. Nuestro objetivo es evaluar la efectividad de nuestras actividades educativas utilizando la red Pharma Phés de un subsidio a la Universidad de BC. La y no es responsable de la formulación o adjudicación de las políticas provinciales de medicamentos. 